\title{
Cross section measurements of the elastic electron - deuteron scattering
}

\author{
Yvonne KohI* \\ for the Al Collaboration \\ Institut für Kernphysik, Johannes Gutenberg-Universität Mainz, \\ Johann-Joachim-Becher-Weg 45, 55128 Mainz \\ E-mail: kohly@uni-mainz.de
}

\begin{abstract}
The electromagnetic form factors of light nuclei provide a sensitive test of our understanding of nuclei. The deuteron in particular, as the only bound two-nucleon system, is a fundamental system that has received extensive attention in the past, by both theory and experiment. Because the deuteron has spin one, three form factors are needed to fully describe the electromagnetic structure of the deuteron. Especially the deuteron charge radius is a favourite observable to compare experiment and calculation. Recently, an extensive measurement campaign has been performed at MAMI (Mainz Microtron) to determine the deuteron charge radius using elastic electron scattering - with the aim to halve the error compared to previous such experiments. The experiment took place at the 3 -spectrometer facility of the A1 Collaboration. Cross section measurements of the elastic electron - deuteron scattering have been performed for 180 different kinematic settings in the low momentum transfer region. From these, the charge form factor can precisely be determined. Fitting the form factor with an appropriate fit function, the radius can then be determined from the slope at zero momentum transfer. The determined radius could then be used as a counterweight to the value obtained from advanced atomic Lamb shift measurements, thus providing additional insight to the proton radius puzzle.
\end{abstract}

53rd International Winter Meeting on Nuclear Physics

26-30 January 2015

Bormio, Italy

${ }^{*}$ Speaker. 


\section{Introduction}

The deuteron holds a key role in hadronic physics. Being a weakly bound state of a proton and a neutron, makes it a unique playground for investigating nuclear forces and nuclear dynamics. Understanding its structure has far-reaching implications, not only for nuclear physics itself but also for the experiments that exploit the deuteron as an effective neutron target in order to obtain information on the neutron. Therefore, it has been extensively studied by both theoreticians and experimentalists. [4]

An important insight into its properties can be obtained by studying the three electromagnetic form factors of the deuteron. The charge form factor is of special interest since it carries information about the charge distribution inside the deuteron and the mean charge radius of the nucleus. Many different experiments have been dedicated to measuring this form factor, but in spite of all the efforts, the charge distribution and the radius are still not understood to a desirable accuracy. Hence a new experiment was underway at MAMI (MAinz MIcrotron), which aimed to measure the deuteron charge form factor in the small $Q^{2}$ region in order to improve the existing results for at least a factor of two and to get a new best nuclear value for the deuteron charge radius. The determined radius could be used as a counterweight to the value obtained from the atomic Lamb shift measurements, thus providing new knowledge on the proton radius puzzle. [5]

\section{Deuteron radius measurements and proton radius}

The proton radius was measured by different kinds of experiments. The results have shown a significant discrepancy between the values for the proton radius, which were obtained by electron scattering measurements and by myonic hydrogen spectroscopy [5]. Because there is no commonly accepted explanation yet, why these two values differ by nearly eight standard deviations this is called the proton radius puzzle.

One possibility to investigate this problem in more detail is to go to the next hydrogen isotope. Also the deuteron radius can be obtained by different kinds of experiments. As you can see in figure 1 the

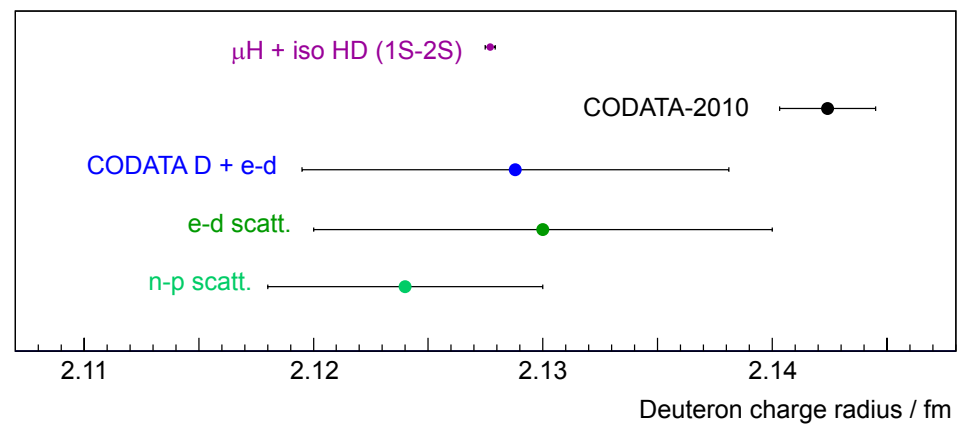

Figure 1: The deuteron radius obtained by different kinds of measurements. See text for details [8].

value obtained by combining the measurements with myonic hydrogen and isoshift measurements (violett point) is known with a very high precision, but it does not agree with the CODATA-2010 
value. One CODATA value (blue point) contains all measurements which were done with deuterium. The difference between this value and the other CODATA value (black point) is that the second one contains also values of the deuteron charge radius which were obtained by measurements with a proton target. However the value from elastic electron - deuteron scattering still has a comparatively large error. In order to distinguish between the CODATA and the combined value a reduction of the $\mathrm{e}-\mathrm{d}$ scattering error of about a factor of two is required.

Also $\mu$ - D measurements were done, but the analysis for these is still ongoing [6].

There have been already several electron deuteron scattering measurements in the past. But as can be seen in figure 2 the results for the cross section do not fully agree. The results from Simon et

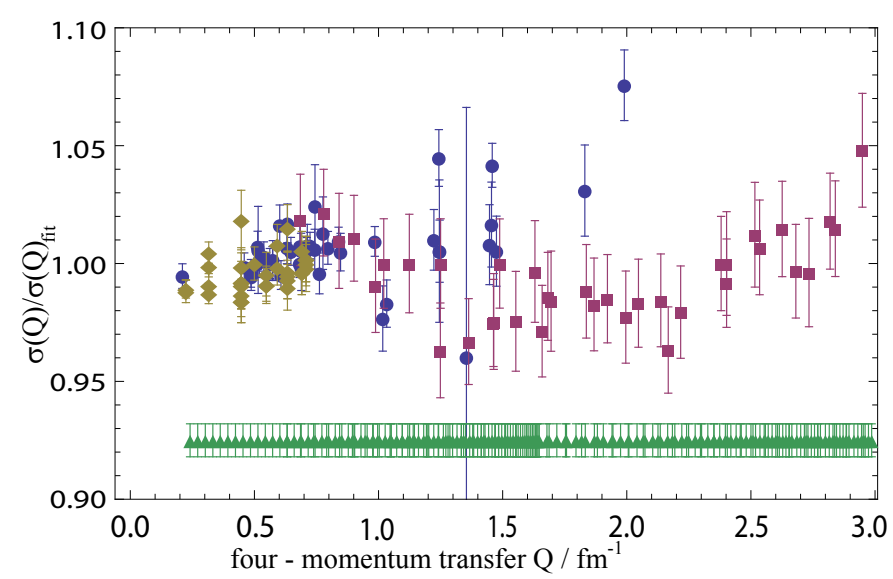

Figure 2: Cross section obtained by prior experiments relatively to fitted result. The green triangles indicate the measured Q range at MAMI with the estimated errors. (Berard et al.:gold, Simon et al.: blue, Platchkov et al.: magenta) $[1,2,3]$

al. indicate a rising slope in the middle of the shown $\mathrm{Q}$ range, but the results from Platchkov et al. indicate a rising slope at higher Q. Also many of the old data points have a large error.

At MAMI we did a measurement with settings over the $\mathrm{Q}$ range indicated by the green triangles in figure 2. The estimated errors for this measurement are smaller than most of the errors of the old data points. The dominating part of these errors are the systematics, the statistic errors are much smaller.

To summarize, the aim of the experiment in Mainz is a high precision determination of the cross section in a wide $\mathrm{Q}$ range to extract the deuteron radius with smaller error than previous determinations of that kind.

\section{Cross section for elastic e - d scattering}

The cross section for unpolarized electron-deuteron scattering can be written in PWIA as [7]:

$$
\frac{d \sigma}{d \Omega}=\frac{d \sigma}{d \Omega}_{\text {Mott }}\left[A\left(Q^{2}\right)+B\left(Q^{2}\right) \tan ^{2}\left(\frac{\theta}{2}\right)\right]
$$


in which

$$
\begin{aligned}
A\left(Q^{2}\right) & =G_{C}^{2}\left(Q^{2}\right)+\frac{8}{9} \eta^{2} G_{Q}^{2}\left(Q^{2}\right)+\frac{2}{3} \eta G_{M}^{2}\left(Q^{2}\right) \\
B\left(Q^{2}\right) & =\frac{4}{3} \eta(1+\eta) G_{M}^{2}\left(Q^{2}\right) \\
\eta & =\frac{Q^{2}}{4 M_{d}}
\end{aligned}
$$

with the charge form factor $G_{C}$, the quadrupol form factor $G_{Q}$, the magnetic form factor $G_{M}$, the mass of the deuteron $M_{d}=1875.612 \mathrm{MeV}$ and the scattering angle of the electron $\theta$.

A notable difference to the cross section of the electron - proton scattering is that in the case of the deuteron (spin 1) there are three form factors. Because of this it is not possible to do a full Rosenbluth-separation.

The charge form factor $G_{C}$ dominates the cross section at low $Q^{2}$. From the slope at $Q^{2}=0$ the deuteron charge radius can be determined:

$$
\left(\frac{d G_{C}}{d Q^{2}}\right)_{Q^{2}=0}=-\frac{1}{6} r_{d}^{2}
$$

The contributions of the other two form factors are small in the low $Q^{2}$ region and will be estimated from a parametrization obtained from dedicated measurements of other groups.

From isotope shift (2S-1S) measurements between hydrogen and deuterium the difference of the quadratic radii is known [5]:

$$
r_{d}^{2}-r_{p}^{2}=3.82007(65) \mathrm{fm}^{2}
$$

With this relation it is possible to get also a value for the proton radius by determination of the deuteron radius.

\section{Experimental setup}

The measurement took place at the MAinz MIcrotron. It is a continuous wave electron accelerator which delivers a high quality beam with beam energies up to $1.6 \mathrm{GeV}$ and beam currents up to $100 \mu \mathrm{A}$. The floor plan of the whole MAMI is shown in figure 3. The electrons are accelerated by three race-track-microtrons (RTM 1-3). For this experiment the beam was then guided directly to the A1-spectrometer hall, because only energies up to $450 \mathrm{MeV}$ were needed.

At the spectrometer hall (figure 4(a)) the electrons are scattered at a cylindrical liquid deuterium cell at the pivot of the spectrometers. The cell walls are made of Havar foil.

The three spectrometers in the hall are quite similar constructed and all can be moved around the pivot. In figure 4(b) the schematics of spectrometer A can be seen in detail.

The scattered electrons enter the spectrometer at the collimator which defines the angular acceptance. They are then guided by different kinds of magnets to the focal plane of the spectrometer. At this point four vertical drift chambers (VDCs) for track reconstruction are located. After the VDCs two layers of scintillators are used for triggering and timing. Finally a threshold Cherenkov detector is installed for particle identification. 


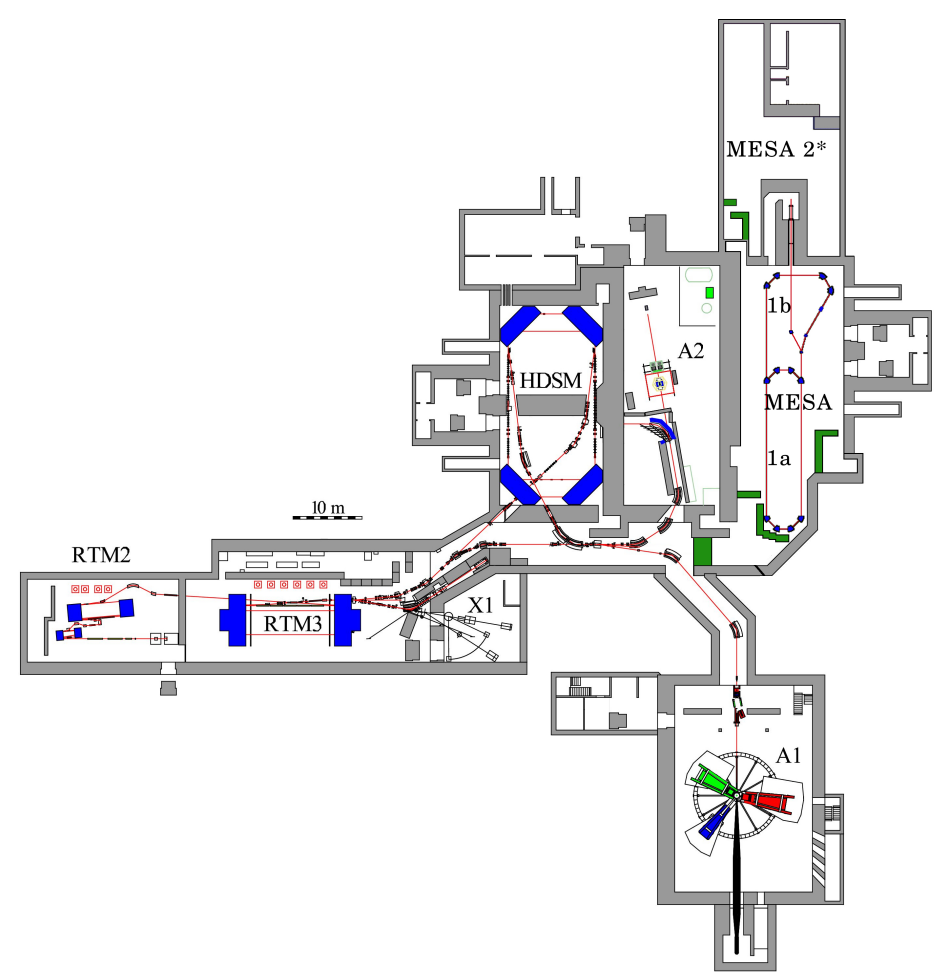

Figure 3: MAMI floor plan. The acceleration process starts on the left side of the shown plan. The electrons are accelerated by three race-track-microtrons (RTM 1-3). For this experiment the beam was then guided directly to the A1-spectrometer hall, because only energies up to $450 \mathrm{MeV}$ were needed. For higher energies the HDSM (harmonic double sided microtron) can be used. On the plan also the other experiment located at MAMI is shown (A2) as well as the planed new parts for the MESA accelerator.

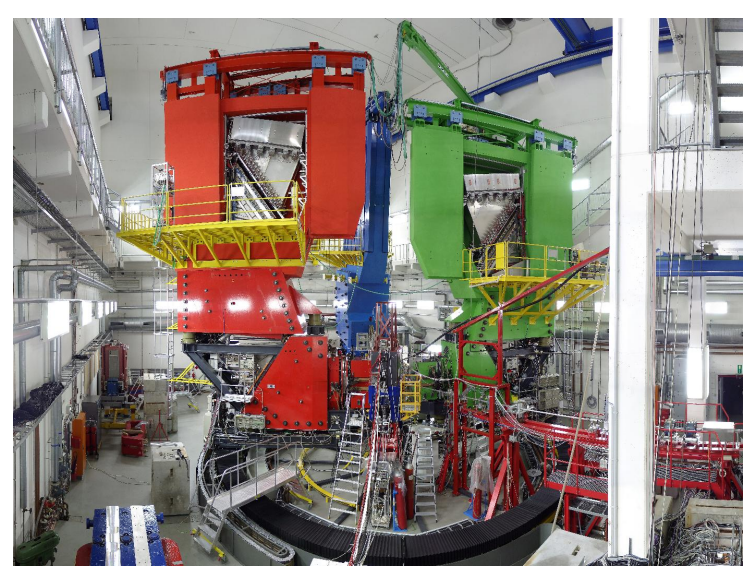

(a)

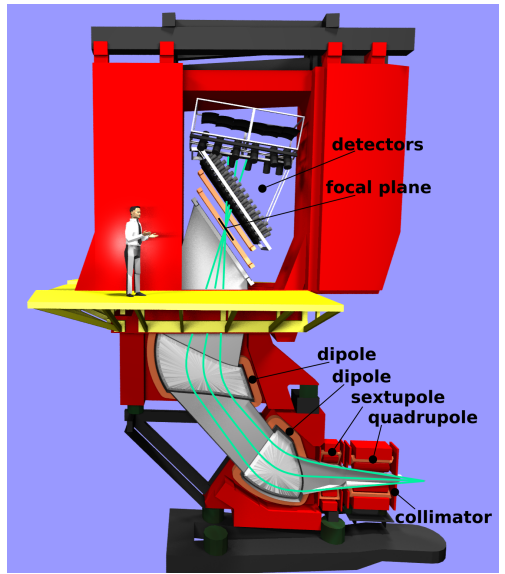

(b)

Figure 4: View into spectrometer hall, target cell is located at the pivot of the spectrometers (a), Spectrometer $\mathrm{A}$ in detail (b), see text for further details. 


\section{Kinematic settings}

The measurement took place in March 2014. In three weeks of beamtime 180 different settings were measured for the deuterium target as well as for corresponding empty cell settings. The empty target has the same size as the deuterium target, but only contains the Havar walls. The settings with this target are necessary for a background subtraction in the analysis. All measured angles for both targets can be seen in figure 5. Data were taken for three different beam energies: 180,

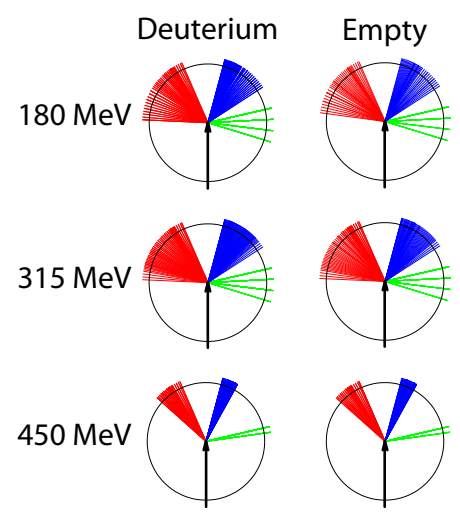

Figure 5: Kinematic settings for deuterium and empty target. The colored lines indicate the measured angles for each spectromter. (spectrometer A: red, spectrometer B: blue, spectrometer C: green).

315 and $450 \mathrm{MeV}$. For all these energies the smallest angle was $15.1^{\circ}$ which was measured by spectrometer B (blue). At the largest measured angle $107^{\circ}$ spectrometer C (green) was located. This spectrometer was mainly used as a luminosity monitor that is why it was set to only a few different angles. Spectrometer A (red) and spectrometer B were moved for each new setting in $1-2^{\circ}$ steps at forward angles.

\section{Analysis of the data}

Starting with the raw data at the three beam energies for both targets, the analysis is performed. The VDC and scintillator data is used for tracking the particles to the focal plane. Well known optics and transfer matrices are then used to reconstruct the particles at the target position.

Auxiliary parts of the analysis include a momentum calibration, efficiency determination and deadtime correction for the detectors.

The event selection is based on the quantity $\Delta \mathrm{E}^{\prime}$, which is the difference between the energy calculated from measured scattering angle and detected energy:

$$
\Delta E^{\prime}=\frac{E}{1-\frac{E}{M_{d}}(1-\cos \theta)}-E^{\prime}
$$

The $\Delta \mathrm{E}$ '-spectra are used to select the elastic line of the electron-deuteron scattering. With this the background subtraction of the target wall contributions as well as the cut around the elastic peak can be done.

In figure 6 a typical measured deuteron $\Delta \mathrm{E}^{\prime}$-spectrum is shown. The elastic line is located around $0 \mathrm{MeV}$. In figure 7 a zoomed in version of the same plot can be seen. Here also other contributions 


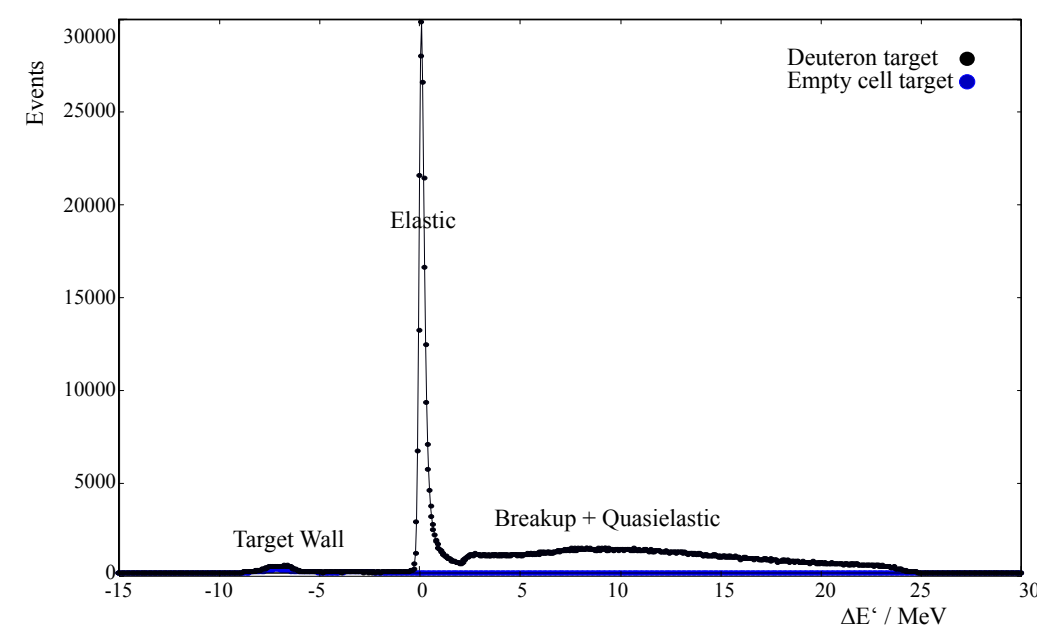

Figure 6: Measured deuteron spectrum at $\mathrm{E}=180 \mathrm{MeV}, \theta_{e}=60.9^{\circ}$. The deuterium data is shown black and the empty cell data in blue.

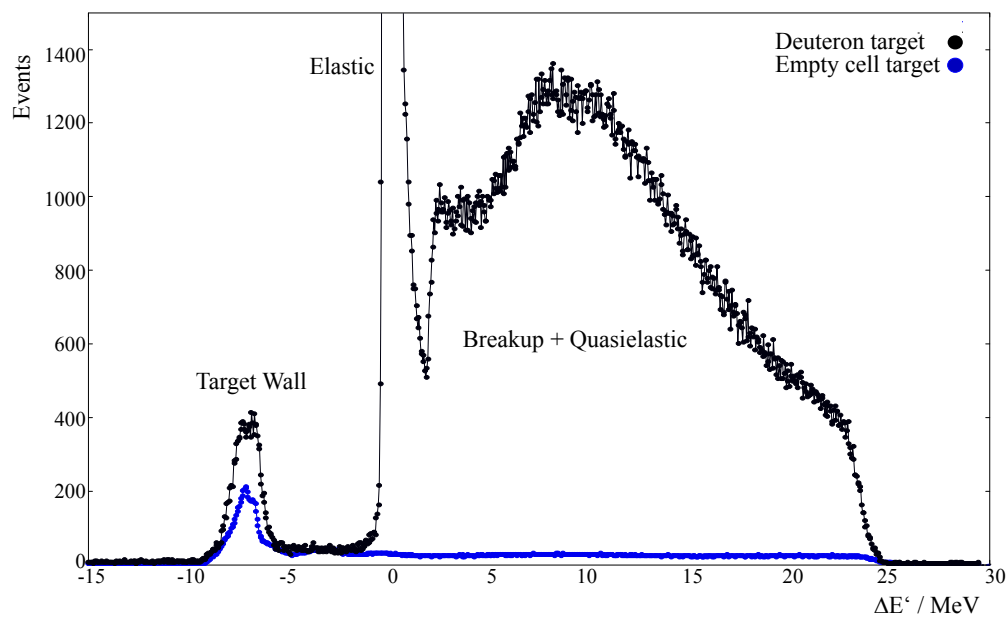

Figure 7: Measured deuteron spectrum at $\mathrm{E}=180 \mathrm{MeV}, \theta_{e}=60.9^{\circ}$. The deuterium data is shown black and the empty cell data in blue.

to the spectrum are clearly visible. At approximately $2 \mathrm{MeV}$ the break up of the deuterium starts as well as the contributions of quasi elastic scattering. That is the reason for a cut at $2 \mathrm{MeV}$ to remove these contributions.

On the other side of the elastic line the contributions of the target wall are resulting in a small peak. To remove the majority of events from the target walls a cut at $-0.5 \mathrm{MeV}$ is performed. For smaller angles the wall peak is closer to the elastic deuterium peak. This makes a correct subtraction of these contributions at the position of the peak very important.

As can be seen in the plot the wall peak in the deuterium data is not just electrons scattered in the wall, since the empty data peak is smaller. This is mostly a result of cryogenic depositions on the target walls in which also scattering takes place. The cryogenic depositions are not yet covered by the simulation. However there is already a simulation for the elastic and quasi-elastic scattering at 
the different nuclei in the Havar-foil. These simulations (blue) and the corresponding data (black) are shown in figure 8 for an energy of $180 \mathrm{MeV}$ and a scattering angle of $60.9^{\circ}$. The simulation of

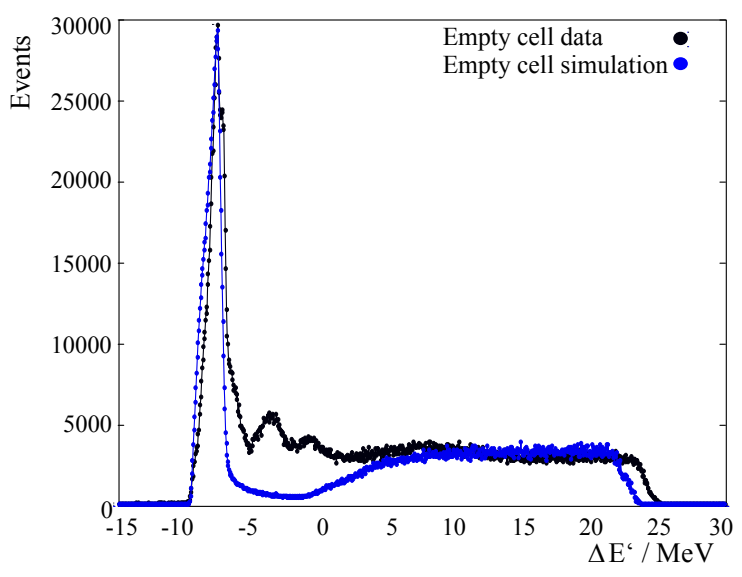

Figure 8: $\Delta \mathrm{E}^{\prime}$-spectrum of empty cell data (black) and result of an empty cell simulation (blue), containing the elastic and quasi-elastic scattering at the Havar walls.

the elastic scattering in the wall mostly contributes to the peak in the plot and the quasi-elastic part becomes mostly manifested in the part of the spectrum at positive $\Delta \mathrm{E}^{\prime}$ 'values.

There are additional peaks located between $-5 \mathrm{MeV}$ and $0 \mathrm{MeV}$, which correspond to excited states of the wall nuclei. The contributions of these excited states are not yet part of the simulation.

In the simulation for the elastic electron - deuteron scattering the energy loss of the electrons in the cell walls are covered as well as the radiative corrections.

\section{Summary and Outlook}

An important insight into the properties of the deuteron can be obtained by studying the three electromagnetic form factors of the deuteron. The charge form factor is of special interest since it carries information about the charge distribution inside the deuteron and the mean charge radius of the nucleus. At Mainz a new experiment dedicated to measure this form factor was performed in 2014. There have been already experiments like this one in the past, but the value from these measurements still has a comparatively large error. That is why the experiment at MAMI aims for a smaller error.

In three weeks of beamtime 180 different settings were measured for the deuterium target as well as for corresponding empty cell settings. These settings contain three different beam energies and scattering angles for the electron between $15.1^{\circ}$ and $107^{\circ}$.

Most of the detector calibrations are finished. The electron - deuteron scattering events are extracted by using cuts on $\Delta \mathrm{E}^{\prime}$ and by using the background subtraction for the target wall contributions in the elastic line.

A validation of the simulation of the target walls has been done and has shown that not all contributions to the target wall spectrum are yet covered by the simulation.

As shown in the previous sections the analysis is still ongoing.

Once the cross sections are extracted the next steps will be to determine the charge form factor, 
from which the charge deuteron radius can be extracted. With the deuteron radius and by using the isotope shift value from 3.6 also the extraction of the proton radius is possible.

\section{References}

[1] R. W. Berard et al. : Phys. Lett. B 47 (1973) 355

[2] G. G. Simon et al. : Nucl. Phys. A 364 (1981) 285

[3] S. Platchkov et al. : Nucl. Phys. A 510 (1990) 740

[4] I. Sick et al. : Prog. Part. Nucl. Phys. 47 (2001) 245

[5] R. Pohl et al. : Nature 466 (2010) 213

[6] P. Indelicato: EINN 2013, Paphos, Cyprus, 2013

[7] D. Abbott et al. : Phys. Rev. Lett. 82 (1999) 1379

[8] P. J. Mohr et al. : Rev. Mod. Phys. 84 (2012) 1527 